\title{
Protective effect of mild hypothermia on oxygen-glucose deprivation injury in rat hippocampal neurons after hypoxia
}

\author{
TIANEN ZHOU ${ }^{1}$, JUN JIANG ${ }^{2}$, MENG ZHANG $^{1}$, YUE FU $^{2}$, ZHENGFEI YANG $^{1}$ and LONGYUAN JIANG ${ }^{1}$ \\ ${ }^{1}$ Department of Emergency, Sun Yat-sen Memorial Hospital of Sun Yat-sen University, Guangzhou, Guangdong 510120; \\ ${ }^{2}$ Department of Emergency, The Affiliated Foshan Hospital of Sun Yat-sen University, Foshan, Guangdong 528000, P.R. China
}

Received November 16, 2012; Accepted March 18, 2013

DOI: $10.3892 / \mathrm{mmr} .2013 .1410$

\begin{abstract}
The present study aimed to establish an oxygen-glucose deprivation (OGD) model of ischemic and hypoxic cerebral neurons to investigate the protective effect of mild hypothermia on neuronal OGD and its mechanisms. OGD injury was significantly mitigated in cells with $24 \mathrm{~h}$ of mild hypothermia compared with cells without mild hypothermia; cell morphology improved, the lactic acid dehydrogenase (LDH) release rate was decreased, cytoactivity was increased and the neuronal apoptotic rate was decreased. By contrast, no significant improvement in injury was observed after $6 \mathrm{~h}$ of mild hypothermia. This suggests that mild hypothermia treatment following OGD is effective only when implemented for $24 \mathrm{~h}$. Additionally, the caspase-3 activity of neurons increased following OGD, which was positively associated with the neuronal apoptotic rate. However, the caspase-3 activity after $24 \mathrm{~h}$ of mild hypothermia was reduced. Simultaneously, the neuronal apoptotic rate was decreased, suggesting that mild hypothermia may inhibit neuronal apoptosis by reducing caspase-3 activity. Therefore, reducing caspase-3 activity potentially constitutes one of the protective mechanisms of mild hypothermia in neuronal OGD.
\end{abstract}

\section{Introduction}

Currently, mild hypothermia is widely used to treat ischemic and hypoxic encephalopathy in a clinical setting. Several studies (1-4) have shown that mild hypothermia inhibits the generation and release of oxygen free radicals and inflammatory reactions following cerebral ischemia and hypoxia, to protect the central nervous system by reducing the oxygen consumption of brain tissues. Previous studies $(5,6)$ have identified

Correspondence to: Professor Longyuan Jiang, Department of Emergency, Sun Yat-sen Memorial Hospital of Sun Yat-sen University, 107 Yanjiangxi Road, Yuexiu, Guangzhou, Guangdong 510120, P.R. China

E-mail: longyuanjiang@yeah.net

Key words: neuron, oxygen-glucose deprivation, mild hypothermia, lactic acid dehydrogenase, release rate, MTT, caspase-3 that mild hypothermia improves nerve function in ischemic and hypoxic encephalopathy by reducing neuronal apoptosis. Apoptosis is closely associated with the caspase protease family. Therefore, the protective effect of mild therapeutic hypothermia on neurons may be associated with the caspase family proteases. Mild hypothermia inhibits caspase-3 activation induced by ischemia-reperfusion injury (7) and reduces $\mathrm{H}_{2} \mathrm{O}_{2}$-induced caspase-3 activity in myocardial cell injury (8). It also reduces caspase- 3 activity in the serum of neonates with ischemic and hypoxic encephalopathy (9). Thus, the protective effect of mild hypothermia treatment on the brain may be achieved by reducing caspase-3 activity following hypoxia and ischemia. However, its protective effect and mechanisms have not yet been fully elucidated. The start time, duration and specific temperature used when applying mild systemic and local hypothermia to the head remain debatable; a limited number of studies have challenged the protective effect of mild hypothermia on the brain (10-14). Therefore, further studies are required to verify the protective effect of mild hypothermia and its mechanism of action.

Studies on the protective effect of mild hypothermia on the brain are divided into in vivo and in vitro trials. In vivo trials usually simulate cerebral ischemia and hypoxia by blocking blood flow to the brain, whereas in vitro trials typically use the current neuronal oxygen-glucose deprivation (OGD) model. In vivo trials have many experimental factors and are characterized by poor control and comparability. Nerve cells cultured in vitro retain the physiological characteristics associated with in vivo neurons and are widely cultured and used to replace in vivo nerve cells in experimental studies. A model of a nerve cell cultured in vitro in hypoxic, sugar-deficient medium has been accepted as a suitable model for simulating brain tissue ischemic injury in vivo. This model is widely used to investigate cerebral ischemic and hypoxic diseases $(15,16)$.

Currently, the majority of studies on the protective effect of mild hypothermia are conducted using OGD and mild hypothermia. However, immediately applying mild hypothermia treatment is difficult during cerebral ischemia and hypoxia. Usually, mild hypothermia treatment is conducted a period of time after cerebral ischemic and hypoxic injury occurrence. To confirm the protective effect of mild hypothermia following cerebral ischemic and hypoxic injury, previous experimental methods must be modified, in which mild hypothermia treatment is performed after OGD. The present study used the 
in vitro experimental method, which applies mild hypothermia after inducing OGD to investigate the protective effect of mild hypothermia on neurons and the potential underlying mechanisms.

\section{Materials and methods}

Cell culture. Approximately $0.1 \mathrm{~g} / 1$ polylysine was used to seal the culture dish for 3 days. Suckling rats aged 1-3 days were provided by the Experimental Animal Center of Sun Yat-sen University (Guangzhou, China). After disinfecting the skin of each rat, the scalp and skull were cut open and brain tissues were immediately removed and placed into a culture dish containing phosphate-buffered saline (PBS). Using an operating microscope, hippocampal tissues were separated and placed in a centrifuge tube containing Dulbecco's modified Eagle's medium (DMEM)/F12 medium (Gibco, Carlsbad, CA, USA) followed by gently sucking in and out using a suction tube. Subsequently, the mixture was centrifuged for $5 \mathrm{~min}$ at $352 \mathrm{x} \mathrm{g}$ and the supernatant was removed. DMEM/F12 containing $10 \%$ fetal bovine serum (FBS; Hyclone, Logan, UT, USA) was added to the remaining precipitate to resuspend the cells. Additionally, trypan blue was used to count the number of active cells. The active cells were inoculated into a culture dish $\left(1 \times 10^{6}\right.$ cells $\left./ \mathrm{ml}\right)$ and incubated in an incubator containing $5 \% \mathrm{CO}_{2}$ at $37^{\circ} \mathrm{C}$. After $24 \mathrm{~h}$, the medium was replaced with a medium containing $2 \%$ B27. On the third day, cytosine arabinoside at a final concentration of $5 \mu \mathrm{mol} / 1$ was added to the cells for $24 \mathrm{~h}$. The culture liquid was replaced once every three days. Neuronal growth and cell morphology were observed. On the 8th day, identification of neuronal microtubule-associated protein 2 (MAP-2) using fluorescence immunohistochemistry was conducted.

Model establishment and groups. An incubator containing three gases was preset to a hypoxic status $\left(37^{\circ} \mathrm{C}, 0.1 \% \mathrm{O}_{2}\right.$, $5 \% \mathrm{CO}_{2}, 94.5 \% \mathrm{~N}_{2}$ ). On the 8th day of culture, the hippocampal neurons and culture medium were removed and $2 \mathrm{ml}$ of sugar-free Earle's liquid $(6.80 \mathrm{~g} \mathrm{NaCl}, 0.40 \mathrm{~g} \mathrm{KCl}, 0.20 \mathrm{~g}$ $\mathrm{CaCl}_{2}, 0.20 \mathrm{~g} \mathrm{MgSO}_{4} \cdot 7 \mathrm{H}_{2} \mathrm{O}, 1.14 \mathrm{~g} \mathrm{NaH}_{2} \mathrm{PO}_{4} \cdot 2 \mathrm{H}_{2} \mathrm{O}$ and $2.20 \mathrm{~g}$ $\mathrm{NaHCO}_{3}$ were dissolved in triple-distilled water to prepare a $1,000-\mathrm{ml}$ solution. The $\mathrm{pH}$ value of the solution was adjusted to 7.4 and a micropore filter used for filtration sterilization) was added, followed by culture in a hypoxic incubator for $2 \mathrm{~h}$. The cells were then collected and the original culture medium was added. The cells were placed into the common incubator $\left(37^{\circ} \mathrm{C}, 19 \% \mathrm{O}_{2}, 5 \% \mathrm{CO}_{2}\right)$ or the mild hypothermic incubator $\left(32^{\circ} \mathrm{C}, 19 \% \mathrm{O}_{2}, 5 \% \mathrm{CO}_{2}\right)$ according to the different groups; the cells were observed and detected after $24 \mathrm{~h}$ of reoxygenation. The experimental cells were randomly divided into 4 groups $(n=6)$; the normal control, simple OGD and two mild hypothermic groups (for $6 \mathrm{~h}$ or $24 \mathrm{~h}$ following OGD). With the exception of the normal control group, the duration of OGD for the groups was $2 \mathrm{~h}$. After OGD, all cells were treated with reoxygenation for $24 \mathrm{~h}$, followed by detection. The present study was carried out in strict accordance with the recommendations in the Guide for the Care and Use of Laboratory Animals of the National Institutes of Health (17). The animal use protocol was reviewed and approved by the Institutional Animal Care and Use Committee (IACUC) of
Sun Yat-sen memorial Hospital of Sun Yat-sen University (Guangzhou, China).

Morphological observation. For the various groups of cells, the cell culture medium was removed following reoxygenation for $24 \mathrm{~h}$. Pre-cooled prefixation liquid at $4^{\circ} \mathrm{C}(2 \%$ glutaric dialdehyde and $2.5 \%$ paraformaldehyde) was added to perform fixation for $15 \mathrm{~min}$. The cells were removed with a cell scraper and centrifuged for $10 \mathrm{~min}$ at a low temperature at 626-1,409 x g. This caused cells in the centrifuge tube to precipitate into a mass. Finally, the cells were stored at $4^{\circ} \mathrm{C}$ and sent to the Electron Microscopy Laboratory in the North Campus of Sun Yat-sen University (Guangzhou, China) for observation.

Lactic acid dehydrogenase $(\mathrm{LDH})$ release rate detection. Following cell injury, cytoplasmic LDH was partially released into the culture medium. A higher LDH release rate indicated more serious cell injury. LDH release rate $(\%)=$ extracellular LDH activity/total cell LDH activity. LDH activity was detected in a 7600-010 full automatic biochemical analyzer (Hitachi, Tokyo, Japan). Firstly, $50 \mu \mathrm{l}$ of the culture medium was used to detect extracellular LDH activity. Then, Triton X-100 lysate was added for cell disruption. The supernatant was collected for detection of LDH activity (18).

MTT assay. The neurons were cultured in a 96-well plate until day 8 . The OGD experimental methods and groups were the same as those mentioned previously. Following reoxygenation for $24 \mathrm{~h}, 20 \mu \mathrm{l}$ of MTT solution at a concentration of $5 \mathrm{mg} / \mathrm{ml}$ (Amresco, Inc., Solon, OH, USA) was added to each well. The cells were incubated at $37^{\circ} \mathrm{C}$ for $4 \mathrm{~h}$. Subsequently, the supernatant was removed and $150 \mu \mathrm{l}$ dimethyl sulfoxide (DMSO; Amresco, Inc.) was added to each well. After the plate was agitated for $10 \mathrm{~min}$, the absorbance of each well was detected at $492 \mathrm{~nm}$ using a microplate reader. The mean of the various wells in each group was considered to be the final result. Cytoactivity $(\%)=$ optical density (OD) of the test group/OD of the normal control group.

Flow cytometry. Following reoxygenation for $24 \mathrm{~h}$, the groups of cells were digested with trypsin, collected and centrifuged. The supernatant was then removed and the cells were resuspended using binding buffer solution. Annexin V-FITC (Bender MedSystems, Vienna, Austria) and propidium iodide (PI; Sigma, St. Louis, MO, USA) were added, followed by shaking. The mixture was allowed to react for $15 \mathrm{~min}$ at room temperature in the dark and a flow cytometer (Becton-Dickinson, Franklin Lakes, NJ, USA) was used to determine the apoptotic rate.

Caspase-3 activity. Caspase-3 is an effector of apoptosis. Apoptosis is more pronounced with increased caspase- 3 activity. The total protein in the neuronal cytoplasm was extracted and then the actual caspase- 3 concentration was detected. Subsequently, $200 \mu \mathrm{g}$ of caspase-3 was collected and treated using the caspase- 3 colorimetric determination reagent kit (BioVision, Inc., Milpitas, CA, USA), according to the manufacturer's instructions. The absorbance at $405 \mathrm{~nm}$ of each well was detected with a microplate reader. Fold increase 


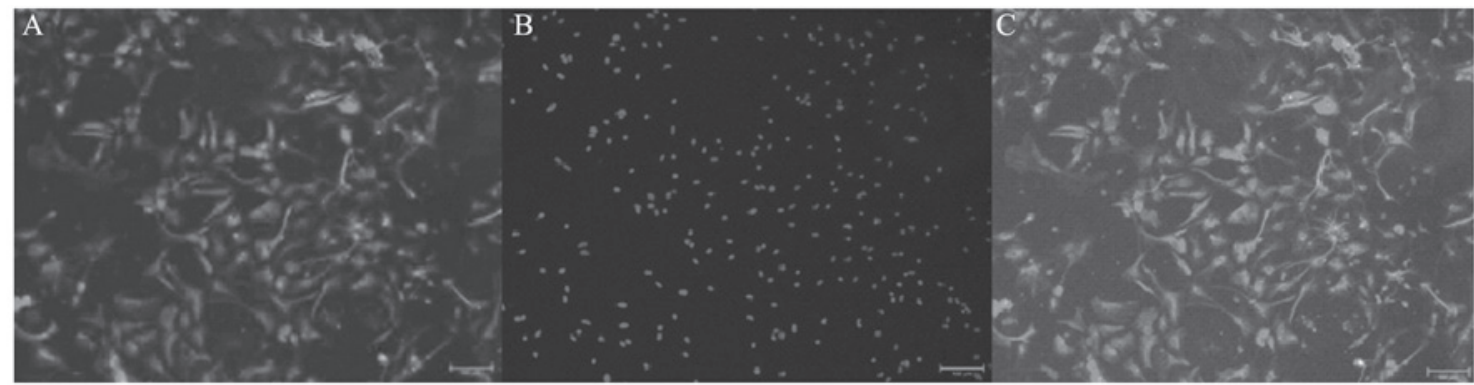

Figure 1. Hippocampal neuron culture and identification. (A) Following MAP-2 immunofluorescence staining, the endochylema and axons of the hippocampal neurons emitted a high level of fluorescence, whereas the nuclei emitted no fluorescence (x200); (B) nonspecific nuclear fluorescence staining with Hoechst 33258 stained all the nuclei (including glial nuclei), whereas the endochylema emitted no fluorescence (x200); (C) after the two images were combined, the hippocampal neurons were revealed to account for $>90 \%$ of the cultured cells (x200). MAP-2, microtubule-associated protein 2.

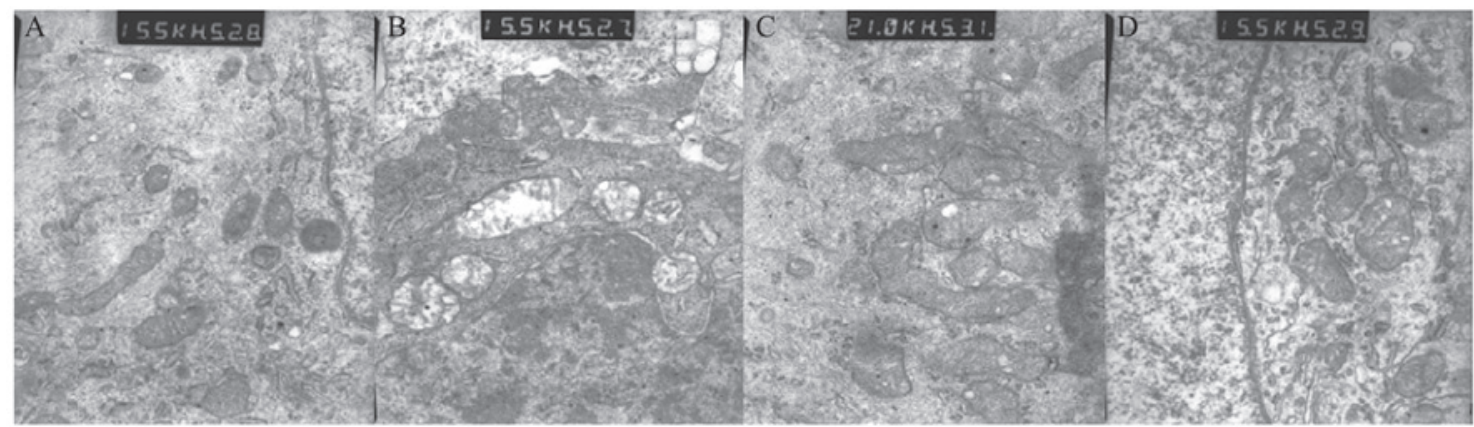

Figure 2. Protective effect of mild hypothermia on neuron morphology. (A) In the normal control group, organelles were integrated and regularly arranged Mitochondria and endoplasmic reticulum were smooth and no edema, cavities or swelling were observed, with clear cell structure (x15,500). (B) In the simple OGD group, the organelles were not integrated, partial organelle degradation was evident and the cell structure was unclear. The mitochondria appeared swollen and cavities were visible (x15,500). (C) In the mild hypothermia for $6 \mathrm{~h}$ following OGD group, the organelles were not integrated and the cell structure was unclear. However, the mitochondria were slightly swollen and cavities were visible (x21,000). (D) In the mild hypothermia for $24 \mathrm{~h}$ following OGD group, the organelles were more integrated and the arrangement was regular. Few cavities were observed in the mitochondria and it was swollen. No edema was observed (x15,500). OGD, oxygen-glucose deprivation.

of caspase activity $=\mathrm{OD}$ of the test group/OD of the normal control group.

Statistical analysis. The measurement data were expressed as the mean \pm standard deviation (SD). A t-test was used to compare the means between samples. A test for the homogeneity of variance was conducted. All the calculations were performed using SPSS 13.0 statistical software. $\mathrm{P}<0.05$ was considered to indicate a statistically significant difference.

\section{Results}

Hippocampal neuron culture and identification results. Following MAP-2 immunofluorescence staining, cortical neurons cultured for 8 days emitted fluorescence when examined under a fluorescence microscope (green light excitation). The endochylema and axons of the hippocampal neurons emitted a high level of fluorescence, whereas the nuclei emitted no fluorescence (Fig. 1A). Nonspecific nuclear fluorescent staining with Hoechst 33258 revealed all the nuclei (including glial nuclei; Fig. 1B). After the two images were combined, the hippocampal neurons were revealed to account for $>90 \%$ of the cultured cells (Fig. 1C).

Protective effect of mild hypothermia on neuronalmorphology. After $24 \mathrm{~h}$ of culture under OGD/reoxygenation, various groups of hippocampal neurons were observed. In the normal control group, the organelles were integrated and regularly arranged. Mitochondria and endoplasmic reticulum were smooth, no edema, cavities or swelling were observed, with clear cell structure (Fig. 2A). In the simple OGD group, the organelles were not integrated and were partially degraded. Their structures were unclear. The mitochondria appeared swollen and more cavities were observed (Fig. 2B). In the group treated with mild hypothermia for $6 \mathrm{~h}$ following OGD, the organelles were not integrated and the cell structure was unclear. However, the mitochondria was slightly swollen and cavities were visible (Fig. $2 \mathrm{C}$ ). In the group treated with mild hypothermia for $24 \mathrm{~h}$ following OGD, the organelles were more integrated and the arrangement was regular. Few cavities were observed in the mitochondria and it was swollen. No edema was observed (Fig. 2D).

Mild hypothermia reduces neuronal $L D H$ release rate. Following OGD, the LDH release rate of nerve cells was markedly increased; the LDH release rate of the simple OGD group was significantly higher compared with that of the normal control group $(\mathrm{P}<0.01)$. No significant difference in $\mathrm{LDH}$ release rate was observed between the mild hypothermia for $6 \mathrm{~h}$ following OGD group and the simple OGD group $(\mathrm{P}>0.05)$. Compared with the simple OGD group, the LDH release rate of the mild hypothermia for $24 \mathrm{~h}$ following OGD 


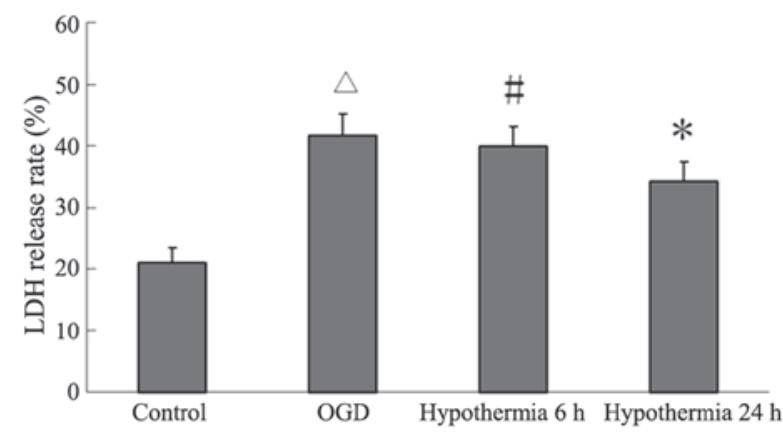

Figure 3. Comparison of the LDH release rates of various groups of neurons. ${ }^{\Delta} \mathrm{P}<0.01$ compared with the normal control group; ${ }^{*} \mathrm{P}>0.05$ compared with the OGD group; ${ }^{*} \mathrm{P}<0.01$ compared with the OGD group. $\mathrm{LDH}$, lactic acid dehydrogenase; OGD, oxygen-glucose deprivation.

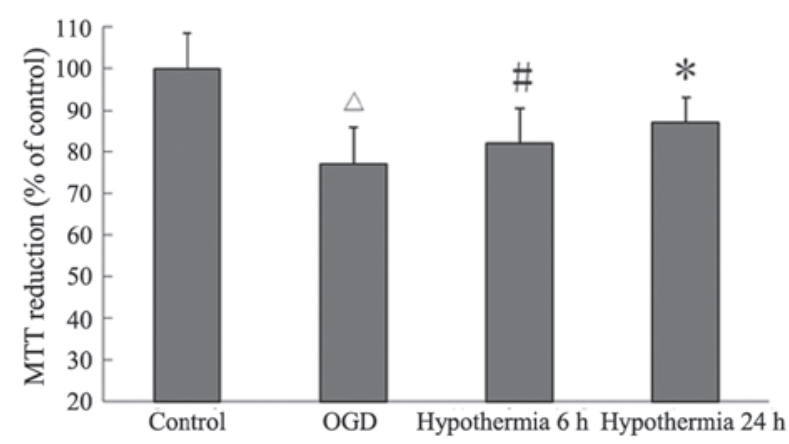

Figure 4. Comparison of the cytoactivity of various groups of neurons (as detected using an MTT assay). ${ }^{\circ} \mathrm{P}<0.01$ compared with the normal control group; ${ }^{\text {P }}>0.05$ compared with the OGD group; ${ }^{*} \mathrm{P}<0.01$ compared with the OGD group. OGD, oxygen-glucose deprivation.

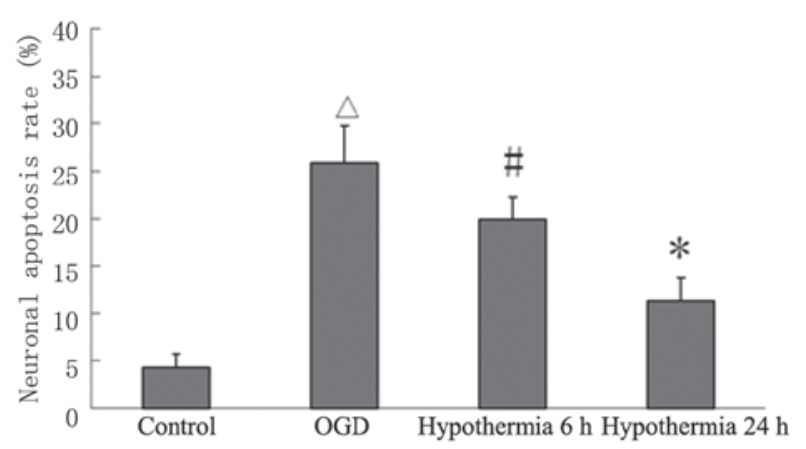

Figure 5. Comparison of the apoptotic rates of the various groups of neurons, as detected by flow cytometry. ${ }^{\triangle} \mathrm{P}<0.01$ compared with the normal control group; ${ }^{\#} \mathrm{P}>0.05$ compared with the OGD group; ${ }^{*} \mathrm{P}<0.01$ compared with the OGD group. OGD, oxygen-glucose deprivation.

group was significantly reduced and the difference was statistically significant $(\mathrm{P}<0.01$; Fig. 3).

Mild hypothermia increases neuronal activity and reduces the apoptotic rate. Following OGD, the cytoactivity of nerve cells was significantly reduced, while the neuronal apoptotic rate significantly increased; the cytoactivity of the simple OGD group was significantly lower compared with that of the normal control group, whereas the neuronal apoptotic rate was

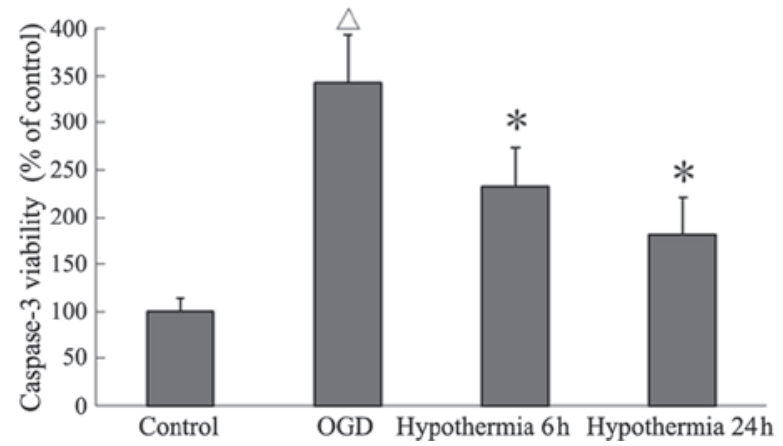

Figure 6. Comparison of the caspase- 3 activity of various groups of neurons. ${ }^{\Delta} \mathrm{P}<0.01$ compared with the normal control group; ${ }^{*} \mathrm{P}<0.01$ compared with the OGD group. OGD, oxygen-glucose deprivation.

significantly higher in the simple OGD group compared with the normal control group $(\mathrm{P}<0.01)$. No significant differences in cytoactivity and neuronal apoptotic rate were observed between the mild hypothermia for $6 \mathrm{~h}$ group and the simple OGD group ( $\mathrm{P}>0.05)$. Compared with the simple OGD group, the cytoactivity of the mild hypothermia for $24 \mathrm{~h}$ group was significantly higher and the neuronal apoptotic rate was significantly reduced. Both differences were statistically significant $(\mathrm{P}<0.01$; Figs. 4 and 5).

Mild hypothermia reduces caspase-3 activity in neuronal cytoplasm. Following OGD, the caspase-3 activity of the nerve cells significantly increased; the caspase-3 activity of the simple OGD group was significantly higher compared with that of the normal control group $(\mathrm{P}<0.01)$. Compared with the simple OGD group, the caspase- 3 activity of the mild hypothermia for $6 \mathrm{~h}$ and the mild hypothermia for $24 \mathrm{~h}$ groups was significantly lower and these differences were statistically significant $(\mathrm{P}<0.05$; Fig. 6).

\section{Discussion}

Nerve cells cultured in vitro retain the relevant physiological characteristics of in vivo neurons. Currently, they are widely cultured to replace in vivo nerve cells in experimental studies (19-22). The in vitro OGD model controls the extracellular environment more simply and accurately than the in vivo model. Therefore, it is often used to investigate changes in biochemistry and cell morphology induced by ischemia and hypoxia and the relevant molecular biology mechanisms (23). Simulating ischemia and hypoxia among in vivo neurons using the OGD model is currently an important research topic and the model is widely used to investigate ischemic and hypoxic encephalopathy (24-27).

Mild hypothermia remains vital to treatment following cerebral ischemic and hypoxic injury. However, the specific methods and details for the application of mild hypothermia have not yet been unified and different viewpoints exist with regard to its curative effect. In clinical research, Yokoyama et al (28) performed a multicenter investigation in Japan which showed that the circulation of 452 patients following cardiopulmonary resuscitation was restored after receiving mild hypothermia treatment for $31.5 \pm 13.9 \mathrm{~h}$. Core temperature was maintained at $33.9 \pm 0.4^{\circ} \mathrm{C}$. Consequently, the 
survival rate of the patients after 30 days was $80.1 \%$, with $55.3 \%$ of the patients retaining good nerve function. This result is significantly higher compared with that of patients who did not receive mild hypothermia treatment in previous studies (29). Walters et al (30) reviewed the previously published literature and identified that after restoring the spontaneous circulation of cardiac arrest patients, mild hypothermia treatment improved nerve function and increased their survival rate. However, large-scale, high-level, multicenter and prospective studies in this area are limited. A number of studies (31) have demonstrated that mild hypothermia treatment improved the nerve function and long-term prognosis of acute spinal cord injury. In a previous study on animals (32), $33^{\circ} \mathrm{C}$ mild hypothermia treatment significantly improved the survival rate of a hemorrhagic shock rat model. Although the treatment disrupted coagulation function to a certain extent, it did not affect the prognosis. Noguchi et al (33) found that mild hypothermia inhibited ischemia-reperfusion injury and arteriolar vasoconstriction in a gerbil model of cerebral ischemia, thereby improving the survival rate in cerebral ischemic injury. Dalen et al (34) demonstrated that mild hypothermia significantly increased the neuronal survival rate following OGD and reduced the generation of inflammatory mediators in an OGD model of nerve cells cultured in vitro. Its protective effect was unrelated to oxygen concentration following reoxygenation. Liu et al (35) showed that mild hypothermia improved cellular metabolism in the OGD model of rat brain sections cultured in vitro, and that earlier hypothermia administration was more effective. As previously mentioned, it is generally considered that mild hypothermia has a protective effect on cerebral ischemic and hypoxic injury. However, a consensus has not been reached and further research is required.

In the present study, the cell injury-associated indicators in the mild hypothermia for $24 \mathrm{~h}$ following OGD group were significantly improved $(\mathrm{P}<0.01)$ and the cell morphology was also significantly improved compared with the simple OGD group. Furthermore, the LDH release rate was significantly reduced, the MTT assay showed that cytoactivity was significantly increased and the flow cytometry assay demonstrated that the neuronal apoptotic rate was significantly reduced. However, the cell injury-associated indicators in the mild hypothermia for $6 \mathrm{~h}$ following OGD group were not significantly improved compared with the simple OGD group ( $\mathrm{P}>0.05)$. Mild hypothermia treatment for $24 \mathrm{~h}$ after OGD markedly relieves neuronal hypoxic and sugar-deficient injury caused by OGD, whereas mild hypothermia treatment for $6 \mathrm{~h}$ did not have a significant protective effect. These results suggest that mild hypothermia treatment after OGD requires application for a long duration, which is in agreement with previously reported research results $(34,36,37)$. Therefore, for ischemic and hypoxic cerebral injury, mild hypothermia treatment for $24 \mathrm{~h}$ is more effective. These results provide an effective theoretical basis for clinical mild hypothermia treatment in ischemic and hypoxic encephalopathy. However, a longer treatment period may not necessarily be better in terms of curative effect due to the potential side-effects of mild hypothermia. Therefore, further studies should be performed to determine the optimal treatment duration.

Mild hypothermia improves nerve function in ischemic and hypoxic encephalopathy by reducing neuronal apoptosis.
Cell apoptosis is closely associated with the caspase protease family. As a cysteine protease family, caspases are critical in apoptosis and are involved in the common pathway of apoptosis. Its members are effector molecules of caspase-3, which implement apoptosis. The period prior to caspase-3 activation is known as the reversible stage of apoptosis and the later period is known as the irreversible stage of apoptosis. Therefore, effectively inhibiting the occurrence and development of apoptosis by reducing caspase-3 activity is possible. Since caspase- 3 is closely associated with apoptosis, numerous studies have utilized it as an indicator for evaluating the curative effects of drugs or methods on cerebral ischemic and hypoxic injury (38-41). Mild hypothermia may achieve its protective effect on the brain by reducing caspase- 3 activity following hypoxia and ischemia.

In the present study, the caspase-3 activity of nerve cells following OGD significantly increased compared with normal control cells $(\mathrm{P}<0.01)$ and was positively associated with an increase in the neuronal apoptotic rate. This suggests that nerve cells may induce programed neuronal cell death following OGD by activating caspase-3, leading to a clear increase in apoptosis. Compared with the simple OGD group, the caspase-3 activity in the mild hypothermia for $24 \mathrm{~h}$ group was significantly decreased $(\mathrm{P}<0.01)$ and the neuronal apoptotic rate was also significantly reduced. This indicates that mild hypothermia may inhibit neuronal apoptosis by reducing caspase-3 activity. Compared with the simple OGD group, the neuronal apoptotic rate in the mild hypothermia for $6 \mathrm{~h}$ group was decreased, but the difference was not significant. However, caspase-3 activity was significantly reduced $(\mathrm{P}<0.05)$. The decrease in caspase-3 activity did not cause a significant reduction in the neuronal apoptotic rate, which is not consistent with the expected results of this study. These results suggest that the apoptotic mechanism is complex and that caspase- 3 is only one of several factors that may affect it. This study showed that a decrease in caspase- 3 activity may be one of the molecular mechanisms for the protective effect of mild hypothermia on the brain. These mechanisms are highly complex and remain to be fully elucidated; thus, further studies are required.

This study was an in vitro trial; however, nerve cells cultured in vitro are not identical to in vivo nerve cells. In vivo neurons are more complex and are widely connected to each other. They also have a lower tolerance for ischemia and hypoxia; thus, they are more easily damaged. Consequently, in vitro experiments are not equivalent to in vivo experiments, and in vitro experiments are unable to replace multicenter clinical studies. The present study provides only a direction and basis for further clinical studies and a model for investigating the relevant mechanisms.

In this study, the duration of mild hypothermia treatment was 6 or $24 \mathrm{~h}$, without examining other time-points. Additionally, mild hypothermia was not applied for $>24 \mathrm{~h}$. For the treatment temperature, only $32^{\circ} \mathrm{C}$ was selected and the effects of other temperatures and comparisons between high-temperature groups were not investigated. Therefore, the present study is not adequately comprehensive. Direction for future studies may include improvement of the present study to obtain a more comprehensive and reliable conclusion. 


\section{References}

1. Bayir H, Clark RS and Kochanek PM: Promising strategies to minimize secondary brain injury after head trauma. Crit Care Med 31 (Suppl 1): S112-S117, 2003.

2. Kinoshita K, Chatzipanteli K, Vitarbo E, Truettner JS, Alonso OF and Dietrich WD: Interleukin-1beta messenger ribonucleic acid and protein levels after fluid-percussion brain injury in rats: importance of injury severity and brain temperature. Neurosurgery 51: 195-203, 2002.

3. Marion DW: Moderate hypothermia in severe head injuries: the present and the future. Curr Opin Crit Care 8: 111-114, 2002.

4. Okauchi M, Kawai N, Nakamura T, Kawanishi M and Nagao S: Effects of mild hypothermia and alkalizing agents on brain injuries in rats with acute subdural hematomas. J Neurotrauma 19 741-751, 2002.

5. Li H and Wang D: Mild hypothermia improves ischemic brain function via attenuating neuronal apoptosis. Brain Res 1368 59-64, 2011.

6. Lin $\mathrm{CH}$, Wu WS, Lin MT, Liu WP, Hsu RB and Chang CP: Attenuating ischemia-induced $\mathrm{H} 9 \mathrm{c} 2$ myoblasts apoptosis by therapeutic hypothermia. Am J Med Sci 339: 258-265, 2010.

7. Kunimatsu T, Kobayashi K, Yamashita A, Yamamoto T and Lee MC: Cerebral reactive oxygen species assessed by electron spin resonance spectroscopy in the initial stage of ischemia-reperfusion are not associated with hypothermic neuroprotection. J Clin Neurosci 18: 545-548, 2011.

8. Diestel A, Drescher C, Miera O, Berger F and Schmitt KR: Hypothermia protects $\mathrm{H} 9 \mathrm{c} 2$ cardiomyocytes from $\mathrm{H}_{2} \mathrm{O}_{2}$ induced apoptosis. Cryobiology 62: 53-61, 2011.

9. Liu CQ, Xia YF, Yuan YX, Li L and Qiu XL: Effects of selective head cooling with mild hypothermia on serum levels of caspase-3 and IL-18 in neonates with hypoxic-ischemic encephalopathy. Zhongguo Dang Dai Er Ke Za Zhi 12: 690-692, 2010 (In Chinese)

10. Brodhun M, Fritz H, Walter B, et al: Immunomorphological sequelae of severe brain injury induced by fluid-percussion in juvenile pigs - effects of mild hypothermia. Acta Neuropathol 101: 424-434, 2001

11. Fritz HG and Bauer R: Secondary injuries in brain trauma: effects of hypothermia. J Neurosurg Anesthesiol 16: 43-52, 2004.

12. Inamasu J, Nakamura Y and Ichikizaki K: Induced hypothemia in experimental traumatic spinal cord injury: an update. J Neurol Sci 209: 55-60, 2003.

13. McIntyre LA, Fergusson DA, Hébert PC, Moher D and Hutchison JS: Prolonged therapeutic hypothemia after traumatic brain injury in adults: a systematic review. JAMA 289: 2992-2999, 2003.

14. Robertson CL, Clark RS, Dixon CE, et al: No long-term benefit from hypothermia after severe traumatic brain injury with secondary insult in rats. Crit Care Med 28: 3218-3223, 2000.

15. Scorziello A, Pellegrini C, Forte L, et al: Differential vulnerability of cortical and cerebellar neurons in primary culture to oxygen glucose deprivation followed by reoxygenation. J Neurosci Res 63: 20-26, 2001.

16. Wang S, Xing Z, Vosler PS, et al: Cellular NAD replenishment confers marked neuroprotection against ischemic cell death: role of enhanced DNA repair. Stroke 39: 2587-2595, 2008.

17. National Research Council: Guide for the Care and Use of Laboratory Animals: 8th Edition. The National Academies press, Washington, DC, pp. 199-200, 2011.

18. Abe K and Matsuki N: Measurement of cellular 3-(4,5-dimethylthiazol-2-yl)-2,5-diphenyltetrazolium bromide (MTT) reduction activity and lactate dehydrogenase release using MTT. Neurosci Res 38: 325-329, 2000.

19. Bai Y, Meng Z, Cui M, et al: An Ang1-Tie2-PI3K axis in neural progenitor cells initiates survival responses against oxygen and glucose deprivation. Neuroscience 160: 371-381, 2009.

20. Labrande C, Velly L, Canolle B, et al: Neuroprotective effects of tacrolimus (FK506) in a model of ischemic cortical cell cultures: role of glutamate uptake and FK506 binding protein $12 \mathrm{kDa}$. Neuroscience 137: 231-239, 2006.

21. Lei Z, Ruan Y, Yang AN and Xu ZC: NMDA receptor mediated dendritic plasticity in cortical cultures after oxygen-glucose deprivation. Neurosci Lett 407: 224-229, 2006.
22. Wang ZF and Tang XC: Huperzine A protects C6 rat glioma cells against oxygen-glucose deprivation-induced injury. FEBS Lett 581: 596-602, 2007.

23. Montero M, Poulsen FR, Noraberg J, et al: Comparison of neuroprotective effects of erythropoietin (EPO) and carbamylerythropoietin (CEPO) against ischemia-like oxygen-glucose deprivation (OGD) and NMDA excitotoxicity in mouse hippocampal slice cultures. Exp Neurol 204: 106-117, 2007.

24. Baltan S, Murphy SP, Danilov CA, Bachleda A and Morrison RS: Histone deacetylase inhibitors preserve white matter structure and function during ischemia by conserving ATP and reducing excitotoxicity. J Neurosci 31: 3990-3999, 2011.

25. Gwak MS, Cao L, Li L and Zuo Z: Isoflurane preconditioning reduces oxygen-glucose deprivation-induced neuronal injury via B-cell lymphoma 2 protein. Environ Toxicol Pharmacol 31: 262-265, 2011

26. Savoia C, Sisalli MJ, Di Renzo G, Annunziato L and Scorziello A: Rosuvastatin-induced neuroprotection in cortical neurons exposed to OGD/reoxygenation is due to nitric oxide inhibition and ERK1/2 pathway activation. Int J Physiol Pathophysiol Pharmacol 3: 57-64, 2011

27. Ziu M, Fletcher L, Rana S, Jimenez DF and Digicaylioglu M: Temporal differences in microRNA expression patterns in astrocytes and neurons after ischemic injury. PLoS One 6: e14724, 2011

28. Yokoyama H, Nagao K, Hase M, et al; J-PULSE-Hypo Investigators: Impact of therapeutic hypothermia in the treatment of patients with out-of-hospital cardiac arrest from the J-PULSE-HYPO study registry. Circ J 75: 1063-1070, 2011.

29. Cheah SO, Ong ME and Chuah MB: An eight year review of exercise-related cardiac arrests. Ann Acad Med Singapore 39: 542-546, 2010.

30. Walters JH, Morley PT and Nolan JP: The role of hypothermia in post-cardiac arrest patients with return of spontaneous circulation: a systematic review. Resuscitation 82: 508-516, 2011.

31. Dietrich WD, Levi AD, Wang M and Green BA: Hypothermic treatment for acute spinal cord injury. Neurotherapeutics 8: 229-239, 2011

32. Iwamoto S, Takasu A and Sakamoto T: Therapeutic mild hypothermia: effects on coagulopathy and survival in a rat hemorrhagic shock model. J Trauma 68: 669-675, 2010.

33. Noguchi K, Matsumoto N, Shiozaki T, et al: Effects of timing and duration of hypothermia on survival in an experimental gerbil model of global ischaemia. Resuscitation 82: 481-486, 2011.

34. Dalen ML, Frøyland E, Saugstad OD, Mollnes TE and Rootwelt T: Post-hypoxic hypothermia is protective in human NT2-N neurons regardless of oxygen concentration during reoxygenation. Brain Res 1259: 80-89, 2009.

35. Liu J, Litt L, Segal MR, Kelly MJ, Yoshihara HA and James TL: Outcome-related metabolomic patterns from $1 \mathrm{H} / 31 \mathrm{P}$ NMR after mild hypothermia treatments of oxygen-glucose deprivation in a neonatal brain slice model of asphyxia. J Cereb Blood Flow Metab 31: 547-559, 2011.

36. Jiang JY, Xu W, Li WP, et al: Effect of long-term mild hypothermia or short-term mild hypothermia on outcome of patients with severe traumatic brain injury. J Cereb Blood Flow Metab 26 771-776, 2006.

37. Shibuta S, Varathan S, Kamibayashi T and Mashimo T: Small temperature variations alter edaravone-induced neuroprotection of cortical cultures exposed to prolonged hypoxic episodes. Br J Anaesth 104: 52-58, 2010.

38. Kunimatsu T, Yamashita A, Kitahama H, Misaki T and Yamamoto T: Measurement of cerebral reactive hyperemia at the initial post-ischemia reperfusion stage under normothermia and moderate hypothermia in rats. J Oral Sci 51: 615-621, 2009.

39. Kuo JR, Lo CJ, Chang CP, Lin HJ, Lin MT and Chio CC: Brain cooling-stimulated angiogenesis and neurogenesis attenuated traumatic brain injury in rats. J Trauma 69: 1467-1472, 2010.

40. Shuja F, Tabbara M, Li Y, et al: Profound hypothermia decreases cardiac apoptosis through Akt survival pathway. J Am Coll Surg 209: 89-99, 2009.

41. Wang Z, Shi Q, Li S, Du J, Liu J and Dai K: Hyperthermia induces platelet apoptosis and glycoprotein Ibalpha ectodomain shedding. Platelets 21: 229-237, 2010. 